\title{
The graphical calculus for ribbon categories: Algebras, modules, Nakayama automorphisms
}

\author{
Jürgen $\mathrm{FUCHS}{ }^{\dagger}$ \\ $\dagger$ Avdelning fysik, Karlstads Universitet, S-65188 Karlstad, Sweden \\ E-mail: jfuchs@fuchs.tekn.kau.se
}

This article is part of the Proceedings of the Baltic-Nordic Workshop, Algebra, Geometry and Mathematical Physics which was held in Tallinn, Estonia, during October 2005.

\begin{abstract}
The graphical description of morphisms in rigid monoidal categories, in particular in ribbon categories, is summarized. It is illustrated with various examples of algebraic structures in such categories, like algebras, (weak) bi-algebras, Frobenius algebras, and modules and bimodules. Nakayama automorphisms of Frobenius algebras are introduced; they are inner iff the algebra is symmetric.
\end{abstract}

\section{Algebras in monoidal categories}

A (unital, associative) algebra is a triple $A=(\dot{A}, m, \eta)$ consisting of a vecor space $\dot{A}$ over some field (or more generally, commutative ring) $\mathbb{k}$, a bilinear map $m: \dot{A} \times \dot{A} \rightarrow \dot{A}$ and an element $e \in \dot{A}$ such that the associativity and unit properties

$$
\begin{array}{ll}
m(m(a, b), c)=m(a, m(b, c)) & \text { for all } a, b, c \in \dot{A} \\
\text { and } \quad m(e, a)=a=m(a, e) & \text { for all } a \in \dot{A}
\end{array}
$$

hold. The datum $\eta$ in the triple $A$ is the linear map from $\mathbb{k}$ to $\dot{A}$ that acts as

$$
\eta(\xi)=\xi e \in \dot{A} \quad \text { for } \quad \xi \in \mathbb{k} .
$$

It is convenient to regard $m$ not as a bilinear map to $\dot{A}$ from the Kronecker product $\dot{A} \times \dot{A}$, but as a linear map to $\dot{A}$ from the tensor product $\dot{A} \otimes_{\mathbb{k}} \dot{A}$. In terms of the linear maps $m$ and $\eta$, the axioms (1.1) of $A$ read

$$
m \circ\left(m \otimes i d_{\dot{A}}\right)=m \circ\left(i d_{\dot{A}} \otimes m\right) \quad \text { and } \quad m \circ\left(\eta \otimes i d_{\dot{A}}\right)=i d_{\dot{A}}=m \circ\left(i d_{\dot{A}} \otimes \eta\right) .
$$

It would actually be more precise to call the structure just described an algebra in the category Vect $_{\mathbb{k}}$ of finite-dimensional $\mathbb{k}$-vector spaces. When this formulation is adopted, the requirement of linearity of the maps $\eta$ and $m$ is already built in automatically, namely by merely demanding that they are allowed maps at all, i.e. that they are morphisms

$$
m \in \operatorname{Hom}(\dot{A} \otimes \dot{A}, \dot{A}) \quad \text { and } \quad \eta \in \operatorname{Hom}(\mathbf{1}, \dot{A})
$$


in $\mathcal{V e c t}_{\mathbb{k}}$. Moreover, there is then no need any longer to refer explicitly to the underlying vector space structure, nor even to elements of $\dot{A}$. Accordingly, this formulation is not only valid for the category $\mathcal{V} e c t_{\mathbb{k}}$, but for any (strict) monoidal category $\mathcal{C}$. That is, we can define a (unital, associative) algebra (or monoid) in $\mathcal{C}$ as a triple $A=(\dot{A}, m, \eta)$ consisting of an object $A$ of $\mathcal{C}$ and of two morphisms (1.4) satisfying the relations (1.3). For general $\mathcal{C}, \otimes$ in (1.4) denotes the (strictly) associative tensor product of $\mathcal{C}$, while $\mathbf{1}$ is the (strict) tensor unit of $\mathcal{C}$ (for $\mathcal{C}=\mathcal{V}$ ect $\mathbb{k}_{\mathbb{k}}$, one has $\otimes=\otimes_{\mathbb{k}}, \mathbf{1}=\mathbb{k}$ and Hom $=$ Hom $_{\mathbb{k}}$ ).

As it turns out, many other concepts of algebra and representation theory can be formulated in the language of monoidal categories, too; see, for instance, [23, 24, 18, 15, $16,19,8,27,7]$. Depending on what additional structure on $A$ is desired, additional properties of the category $\mathcal{C}$ can be needed. Let me give a few examples:

- A left $A$-module over an algebra $A$ in $\mathcal{C}$ is a pair $M=(\dot{M}, \rho)$ consisting of an object $\dot{M}$ of $\mathcal{C}$ and a morphism $\rho \in \operatorname{Hom}(\dot{A} \otimes \dot{M}, \dot{M})$ satisfying the representation properties

$$
\rho \circ\left(i d_{\dot{A}} \otimes \rho\right)=\rho \circ\left(m \otimes i d_{\dot{M}}\right) \quad \text { and } \quad \rho \circ\left(\eta \otimes i d_{\dot{M}}\right)=i d_{\dot{M}} .
$$

Analogously, a right $A$-module is a pair $(\dot{M}, \varrho)$ with $\varrho \in \operatorname{Hom}(\dot{M} \otimes \dot{A}, \dot{M})$ satisfying $\varrho \circ\left(\varrho \otimes i d_{\dot{A}}\right)=\varrho \circ\left(i d_{\dot{M}} \otimes m\right)$ and $\varrho \circ\left(i d_{\dot{M}} \otimes \eta\right)=i d_{\dot{M}}$.

And an $A$-bimodule is a triple $(\dot{M}, \rho, \varrho)$ such that $(\dot{M}, \rho)$ is a left $A$-module and $(\dot{M}, \varrho)$ a right $A$-module, with commuting left and right actions of $A$, i.e.

$$
\rho \circ\left(i d_{\dot{A}} \otimes \varrho\right)=\varrho \circ\left(\rho \otimes i d_{\dot{A}}\right) .
$$

An intertwiner between (left, say) $A$-modules $M=(\dot{M}, \rho)$ and $M^{\prime}=\left(\dot{M}^{\prime}, \rho^{\prime}\right)$ is a morphism $f \in \operatorname{Hom}\left(\dot{M}, \dot{M}^{\prime}\right)$ that intertwines the $A$-action on $M$ and $M^{\prime}$ in the sense that $f \circ \rho=\rho^{\prime} \circ\left(i_{\dot{A}} \otimes f\right)$. The category $\mathcal{C}_{A \mid}$ of left $A$-modules has by definition the left $A$-modules as objects and their intertwiners as morphisms. Analogously there is a category $\mathcal{C}_{\mid A}$ of right $A$-modules, as well as a category $\mathcal{C}_{A \mid A}$ of $A$-bimodules; the latter is again monoidal.

- A co-algebra in $\mathcal{C}$ is a triple $C=(\dot{C}, \Delta, \varepsilon)$ where $\dot{C}$ is an object of $\mathcal{C}$ and the coproduct $\Delta$ and counit $\varepsilon$ are morphisms with properties dual to the properties (1.3) of the product and unit, i.e. $\Delta \in \operatorname{Hom}(\dot{A}, \dot{A} \otimes \dot{A})$ and $\varepsilon \in \operatorname{Hom}(\dot{A}, \mathbf{1})$ obey

$$
\left(\Delta \otimes \mathrm{id}_{\dot{A}}\right) \circ \Delta=\left(i d_{\dot{A}} \otimes \Delta\right) \circ \Delta \quad \text { and } \quad\left(\varepsilon \otimes \mathrm{id}_{\dot{A}}\right) \circ \Delta=\mathrm{id}_{\dot{A}}=\left(i d_{\dot{A}} \otimes \varepsilon\right) \circ \Delta .
$$

Similarly, a left $C$-comodule is a pair $(\dot{M}, \delta)$ with $\delta \in \operatorname{Hom}(\dot{M}, \dot{C} \otimes \dot{M})$ obeying relations dual to (1.5). Analogously one defines right $C$-comodules and $C$-bicomodules.

- The induced left $A$-module $\operatorname{Ind}_{A}(U)$ is given, for any object $U$ of $\mathcal{C}$, by

$$
\operatorname{Ind}_{A}(U)=\left(\dot{A} \otimes U, m \otimes i d_{U}\right) .
$$

Every induced module is projective, and every simple $A$-module (i.e., simple object of $\mathcal{C}_{A \mid}$ ) is a quotient of an induced module [22].

Analogously there are the notions of induced right module, and of induced bimodule. The underlying objects of the latter bimodules are of the form $\dot{A} \otimes U \otimes \dot{A}$. If $\mathcal{C}$ is a braided monoidal category, then there are interesting bimodule structures on the objects $\dot{A} \otimes U$ as well. These are known as $\alpha^{ \pm}$-induced bimodules $\alpha_{A}^{ \pm}(U)=\left(\dot{A} \otimes U, \rho, \varrho^{ \pm}\right)$; the left action $\rho$ of $A$ is as in (1.8), while the right action is given by

$$
\varrho^{+}=\left(m \otimes \mathrm{id}_{U}\right) \circ\left(i d_{\dot{A}} \otimes c_{U, \dot{A}}\right) \quad \text { and } \quad \varrho^{-}=\left(m \otimes i d_{U}\right) \circ\left(i d_{\dot{A}} \otimes\left(c_{\dot{A}, U}\right)^{-1}\right),
$$


respectively. Here we denote by $c_{U, V} \in \operatorname{Hom}(U \otimes V, V \otimes U)$ the braiding isomorphisms of $\mathcal{C}$.

- For braided $\mathcal{C}$ there is also the notion of a (left) $A$-dimodule over an algebra $A$ in $\mathcal{C}$ that is also a coalgebra. This is a triple $(\dot{M}, \rho, \delta)$ such that $(\dot{M}, \rho)$ is a (left) $A$-module and $(\dot{M}, \delta)$ a (left) $A$-comodule and such that the action and coaction of $A$ commute, i.e.

$$
\delta \circ \rho=\left(\mathrm{id}_{\dot{A}} \otimes \rho\right) \circ\left(c_{\dot{A}, \dot{A}} \otimes \mathrm{id}_{\dot{M}}\right) \circ\left(\mathrm{id}_{\dot{A}} \otimes \delta\right) .
$$

Note that there is also another, equally natural, notion of $A$-dimodule, obtained from the one just defined by replacing the self-braiding $c_{\dot{A}, \dot{A}}$ of $\dot{A}$ by its inverse. The two structures coincide iff $c_{\dot{A}, \dot{A}}$ squares to $i_{\dot{A}}$, and thus in particular if the braiding of $\mathcal{C}$ is actually a symmetry, as is e.g. the case for $\mathcal{C}=\mathcal{V} e c t_{\mathbb{k}}$.

- Assume still that $\mathcal{C}$ is braided. Then a bi-algebra in $\mathcal{C}$ is an algebra that is also a coalgebra in such a way that the coproduct and counit are morphisms of algebras, while the unit, and automatically also the product, are coalgebra morphisms. Thus a bi-algebra is a quintuple $(\dot{H}, m, \eta, \Delta, \varepsilon)$ such that $(\dot{H}, m, \eta)$ is an algebra, $(\dot{H}, \Delta, \varepsilon)$ is a co-algebra, and

$$
\begin{aligned}
& (m \otimes m) \circ\left(\mathrm{id}_{\dot{A}} \otimes c_{A, A} \otimes i d_{\dot{A}}\right) \circ(\Delta \otimes \Delta)=\Delta \circ m \\
& \text { and } \quad \varepsilon \circ m=\varepsilon \otimes \varepsilon, \quad \Delta \circ \eta=\eta \otimes \eta .
\end{aligned}
$$

(Again we have made one out of two equally sensible choices for the braiding. They correspond to two equally natural definitions of algebra structure on the object $\dot{A} \otimes \dot{A}$.)

Further, a Hopf algebra $H=(\dot{H}, m, \eta, \Delta, \varepsilon, \mathrm{s})$ in $\mathcal{C}$ is a bi-algebra with an additional morphism $\mathrm{s} \in \operatorname{Hom}(\dot{H}, \dot{H})$, the antipode, which is required to be an inverse to the identity morphism id $\dot{H}_{\dot{H}}$ with respect to the convolution product that is defined as $\alpha * \beta:=m \circ(\alpha \otimes \beta) \circ \Delta$ for $\alpha, \beta \in \operatorname{Hom}(\dot{H}, \dot{H})$.

\section{Graphical calculus}

For presenting further examples, it is stimulating to resort to the geometry of tensor calculus [12], i.e. introduce a graphical notation for morphisms in a strict monoidal category. This formalism is most convenient for visualizing algebraic structures like those introduced above, see e.g. $[15,4,16,8,7]$. Often it is even indispensible in that it allows for graphical proofs that are easier, and more illuminating, than when using ordinary formulas. It has also been instrumental for recent progress in the understanding of two-dimensional conformal quantum field theory $[2,4,16,8,10,5]$. While the graphical calculus is only applicable to strict monoidal categories, this restriction is inessential. Indeed, by the coherence theorems [17] every monoidal category is equivalent to a strict one, and this extends to categories with additional structure, see e.g. [21]. In most applications the monoidal category of interest can safely be replaced with the equivalent strict monoidal category.

We draw identity morphisms $i d_{U}$ as vertical lines (often as straight lines, but local deformations like bending of a line do not change the meaning of a graph) labeled $U$, and a general morphism $f \in \operatorname{Hom}(U, V)$ as a small coupon with an incoming (i.e., entering the lower edge of the coupon) line labeled $U$ and an outgoing line labeled $V$. For particular 
morphisms that appear often we use more specific notation, e.g. we write
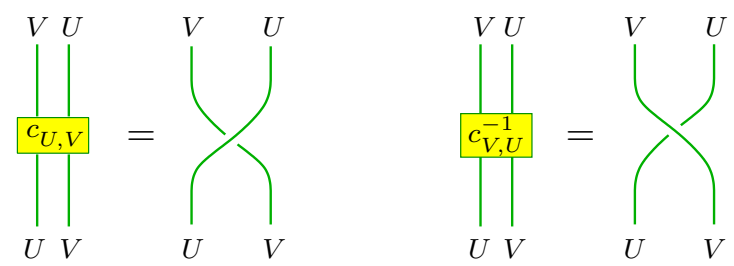

for the braiding isomorphisms $c_{U, V} \in \operatorname{Hom}(U \otimes V, V \otimes U)$ and their inverses, and denote the (co)product and (co)unit morphisms of a (co)algebra by<smiles>C#CC(I)CI</smiles><smiles>[AlH]C[C@@H](I)C[Tl]</smiles>

With these notations e.g. the defining properties (1.3) of the product and unit morphisms look as follows:
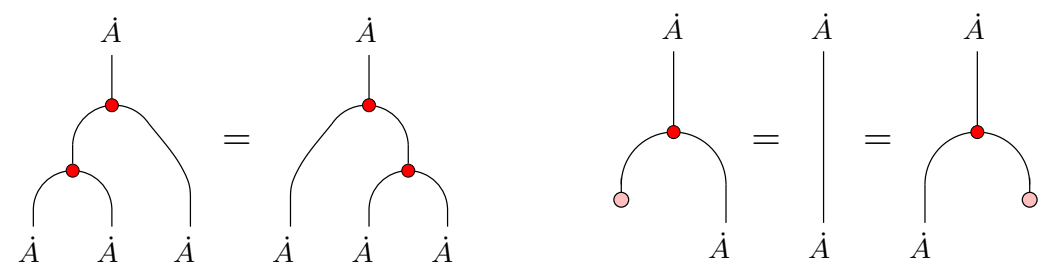

An example involving a braiding is given by the bi-algebra axioms (1.11) and (1.12), which look like
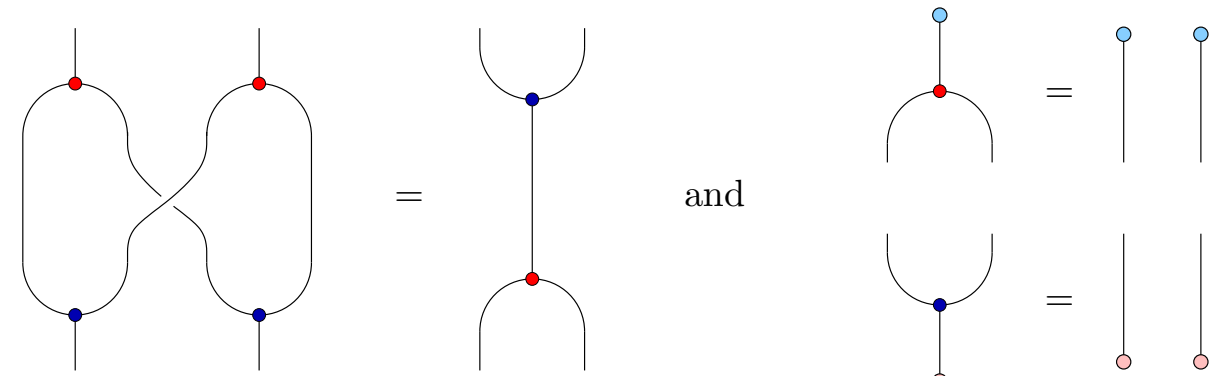

and

respectively. (Here and in the sequel we suppress the labels of the lines when they are obvious from the context.)

As another illustration, consider a weak bi-algebra [25] in a braided monoidal category $\mathcal{C}$. This is a quintuple $(\dot{H}, m, \eta, \Delta, \varepsilon)$ for which $(\dot{H}, m, \eta)$ is an algebra, $(\dot{H}, \Delta, \varepsilon)$ is a co-algebra, and the bi-algebra axiom (1.11) holds, while (1.12) is replaced by the weaker 
properties

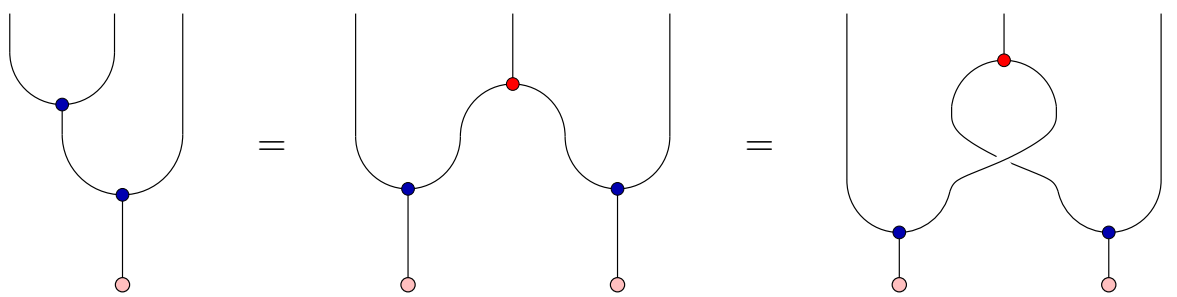

and
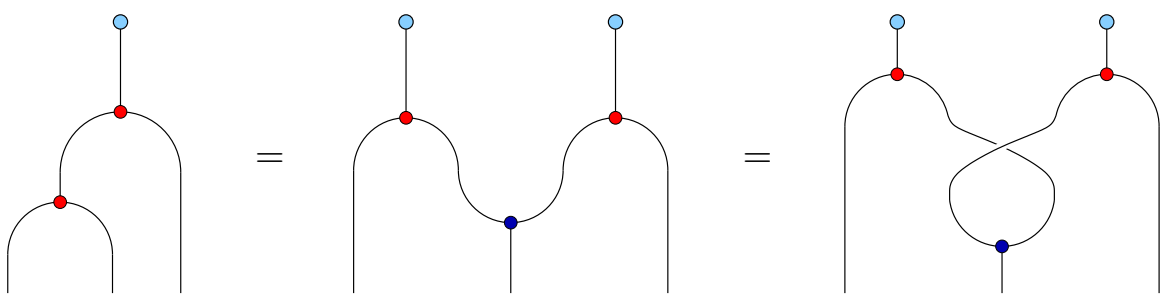

It follows e.g. that
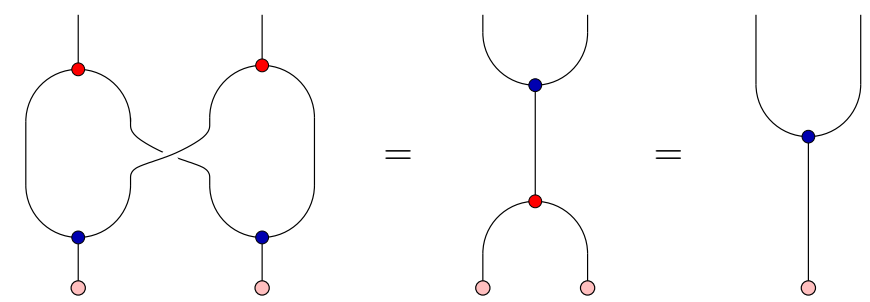

which is the version in $\mathcal{C}$ of the idempotent property of $\Delta(e) \cdot \Delta(e)=\Delta(e)$ that is valid for a weak bi-algebra in $\mathcal{V} e c t_{\mathbb{k}}$.

Every bi-algebra is in particular a weak bi-algebra; the expressions in (2.5) then all reduce to $\varepsilon \otimes \varepsilon \otimes \varepsilon$ and to $\eta \otimes \eta \otimes \eta$, respectively.

\section{Frobenius algebras and ribbon categories}

There are several equivalent definitions of a Frobenius algebra in $\mathcal{V}_{\text {ect }}$, and similarly there are several possibilities to characterize Frobenius algebras in a monoidal category $\mathcal{C}$. The following one does not require any further structure on $\mathcal{C}$ : A Frobenius algebra in $\mathcal{C}$ is a quintuple $A=(\dot{A}, m, \eta, \Delta, \varepsilon)$ such that $(\dot{A}, m, \eta)$ is an algebra, $(\dot{A}, \Delta, \varepsilon)$ is a co-algebra, and $\Delta$ is a morphism of $A$-bimodules. That in the case of $\mathcal{V}_{\text {ect }}$ (3.1) is equivalent to the usual definition is e.g. shown in [1]. In pictures, the bimodule morphism property of $\Delta$ reads

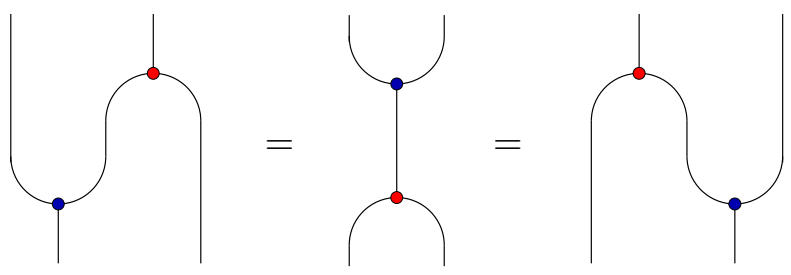

Unlike for the bi-algebra axiom (1.11) no braiding is involved in (3.1). Also note that here we use the same symbols for the coproduct and counit as in the case of bi- or Hopf algebras above, even though now neither $\Delta$ nor $\varepsilon$ is an algebra morphism. 
A large number of nontrivial examples of Frobenius algebras is provided by so-called Schellekens algebras $[9,6]$; as objects they are direct sums of invertible objects, and they are classified in terms of the cohomology of the Picard group (the group of isomorphism classes of invertible objects) of $\mathcal{C}$ and of its subgroups. For a Frobenius algebra, any left module $(\dot{M}, \rho)$ gives rise to a left comodule (and vice versa), namely $(\dot{M}, \varrho)$ with $\varrho:=\left(\mathrm{id}_{\dot{A}} \otimes \rho\right) \circ\left((\Delta \circ \eta) \otimes i d_{\dot{M}}\right)$. This gives rise to an $A$-dimodule iff $A$ is commutative, i.e. $m \circ c_{\dot{A}, \dot{A}}=m$ for a braiding $c_{\dot{A}, \dot{A}}$ in $\mathcal{C}$.

In Vect $_{\mathbb{k}}$, a conventional definition of the Frobenius property is that $A$ is isomorphic as a left (or, equivalently, as a right) $A$-module to $\dot{A}^{\vee}=\operatorname{Hom}_{\mathbb{k}}(\dot{A}, \mathbb{k})$ (see e.g. $[3,13,20]$ ). To be able to formulate this isomorphism property in other monoidal categories $\mathcal{C}$ we need a notion of dual object. It is actually natural to require $\mathcal{C}$ to be sovereign, i.e. that there exist left and right duality functors that coincide both on objects and on morphisms. That is, any object $U$ of $\mathcal{C}$ has a left and right dual object $U^{\vee}$ and there are left and right evaluation morphisms $d_{U} \in \operatorname{Hom}\left(U^{\vee} \otimes U, \mathbf{1}\right), \tilde{d}_{U} \in \operatorname{Hom}\left(U \otimes U^{\vee}, \mathbf{1}\right)$, as well as left and right coevaluation morphisms $b_{U} \in \operatorname{Hom}\left(\mathbf{1}, U \otimes U^{\vee}\right), \tilde{b}_{U} \in \operatorname{Hom}\left(\mathbf{1}, U^{\vee} \otimes U\right)$, satisfying

$$
\begin{array}{ll}
\left(i d_{U} \otimes d_{U}\right) \circ\left(b_{U} \otimes i d_{U}\right)=i d_{U}, & \left(d_{U} \otimes i d_{U}\right) \circ\left(i d_{U^{\vee}} \otimes b_{U}\right)=i d_{U^{\vee}} \\
\left(\tilde{d}_{U} \otimes i d_{U}\right) \circ\left(i d_{U} \otimes \tilde{b}_{U}\right)=i d_{U}, & \left(i d_{U} \otimes \tilde{d}_{U}\right) \circ\left(\tilde{b}_{U} \otimes i d_{U}\right)=i d_{U} \vee
\end{array}
$$

as well as

$$
\begin{aligned}
\left(d_{V} \otimes i d_{U^{\vee}}\right) \circ\left(i d_{V^{\vee}} \otimes f \otimes i d_{U^{\vee}}\right) \circ\left(i d_{V^{\vee}} \otimes b_{U}\right) \\
=\left(i d_{U^{\vee}} \otimes \tilde{d}_{V}\right) \circ\left(i d_{U^{\vee}} \otimes f \otimes i d_{V^{\vee}}\right) \circ\left(\tilde{b}_{U} \otimes i d_{V^{\vee}}\right)
\end{aligned}
$$

for any $f \in \operatorname{Hom}(U, V)$. We draw the evaluation and coevaluation morphisms as
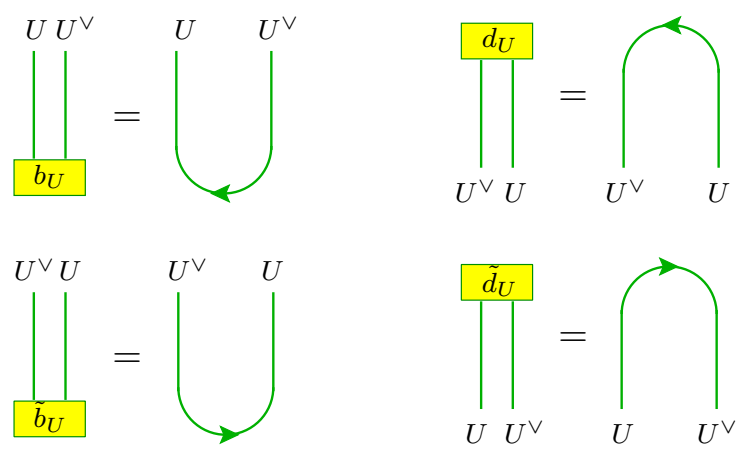

The object $\dot{A}^{\vee}$ can be naturally endowed with left and right $A$-module structures by
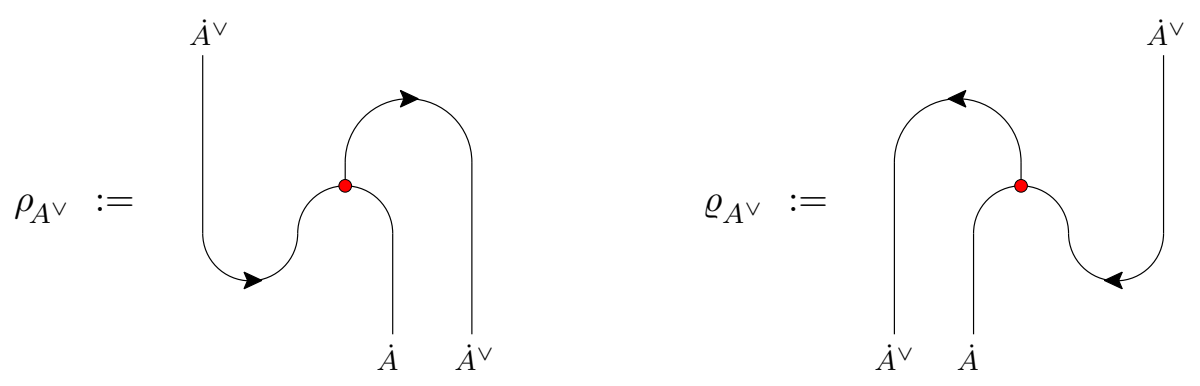
Now the Frobenius property (3.1) and the duality axioms (3.2) imply that the morphisms
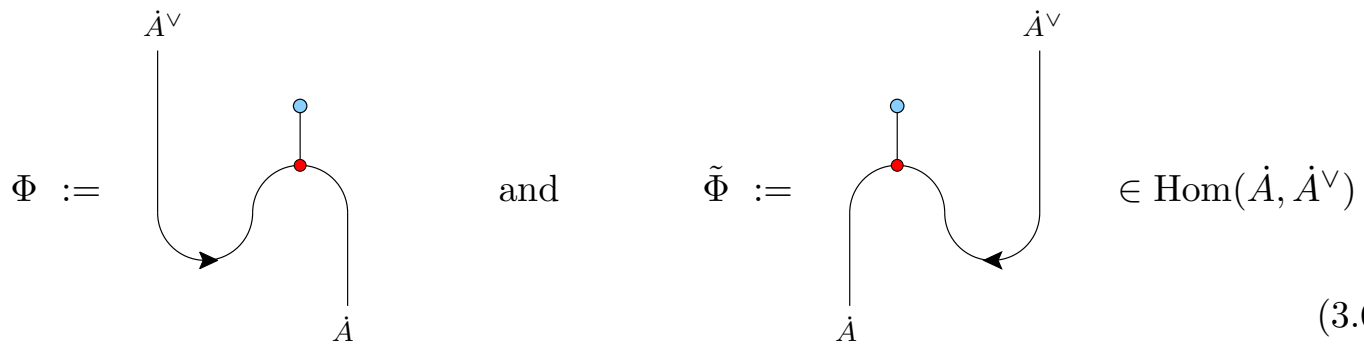

are invertible, with inverses $\Phi^{-1}=\left(i d_{\dot{A}} \otimes \tilde{d}_{A}\right) \circ\left((\Delta \circ \eta) \otimes i d_{\dot{A}^{\vee}}\right)$ and $\tilde{\Phi}^{-1}=\left(d_{A} \otimes i d_{\dot{A}}\right) \circ$ $\left(\right.$ id $\left._{\dot{A}^{\vee}} \otimes(\Delta \circ \eta)\right)$, respectively. Moreover, one can check that $\Phi$ and $\tilde{\Phi}$ intertwine, respectively, the left and right $A$-module structures on $\dot{A}$ (given by $\rho_{A}=m=\varrho_{A}$ ) and on $\dot{A}^{\vee}$.

Conversely, given an algebra $A$ and an isomorphism $\Phi$ (or $\tilde{\Phi}$ ) of $\dot{A}$ and $\dot{A}^{\vee}$ as left (or right) $A$-modules, one may define a coproduct on $A$ by

$$
\Delta:=\left(\Phi^{-1} \otimes m\right) \circ\left(\tilde{b}_{A} \otimes i d_{\dot{A}}\right)
$$

(respectively, as $\left.\left(m \otimes \tilde{\Phi}^{-1}\right) \circ\left(i d_{\dot{A}} \otimes \tilde{b}_{A}\right)\right)$, and then coassociativity of $\Delta$ follows from the intertwining property combined with associativity of $m$. Thus in a sovereign monoidal category the Frobenius axiom (3.1) is indeed equivalent to the existence of isomorphic $A$-module intertwiners (3.6).

The analogue of the nondegenerate invariant bilinear form, whose existence constitutes another (convenient [14]) definition of a Frobenius algebra in $\mathcal{V}_{e c t}$, is the morphism

$$
\tilde{d} \circ\left(\mathrm{id}_{\dot{A}} \otimes \Phi\right)=d \circ\left(\tilde{\Phi} \otimes \mathrm{id}_{\dot{A}}\right) \in \operatorname{Hom}(\dot{A} \otimes \dot{A}, \mathbf{1}) .
$$

With $\Phi$ and $\tilde{\Phi}$ defined as in (3.6), this is nothing but $\varepsilon \circ \Delta$. Conversely, if one regards (3.8) as defining the analogue of a bilinear form, then requiring it to be nondegenerate amounts to $\Phi$ and $\tilde{\Phi}$ being invertible, while requiring it to be invariant amounts to $\Phi$ and $\tilde{\Phi}$ being intertwiners of left and right $A$-modules, respectively.

In a sovereign monoidal category every object is isomorphic to its bidual, via the isomorphism $\left(\mathrm{id}_{U^{\vee \vee}} \otimes d_{U}\right) \circ\left(\tilde{b}_{U} \otimes \mathrm{id}_{U}\right) \in \operatorname{Hom}\left(U, U^{\vee \vee}\right)$. In a sovereign monoidal category that is also braided, the left and right dualities can be combined with the braiding to define twist isomorphisms $\theta_{U}$. The defining properties of braiding, right (or left) duality and twist are precisely those for ribbons in three-space projected nonsingularly to the plane; accordingly, braided sovereign monoidal categories are also called ribbon categories [2]. In a ribbon category, the above isomorphisms in $\operatorname{Hom}\left(U, U^{\vee \vee}\right)$, combined with the twist isomorphisms, furnish a natural equivalence between the bidual functor ? ${ }^{\vee V}$ and the identity functor.

\section{Nakayama automorphisms and symmetric algebras}

Let $A$ be a Frobenius algebra in a sovereign monoidal category $\mathcal{C}$. The endomorphism

$$
\omega_{\mathrm{N}}:=\tilde{\Phi}^{-1} \circ \Phi
$$


in $\operatorname{Hom}(\dot{A}, \dot{A})$, with $\Phi$ and $\tilde{\Phi}$ as in (3.6), is clearly an isomorphism, with inverse $\Phi^{-1} \circ \tilde{\Phi}$. Moreover, one has the

Proposition: The morphism (4.1) is an algebra automorphism of $A$.

Proof. Using successively the Frobenius property, coassociativity, sovereignty of $\mathcal{C}$ (applied to the coproduct that is marked by a dashed box in the second picture below) together with the duality axioms (3.2), then again the Frobenius property, and finally associativity, one derives the following chain of equalities:

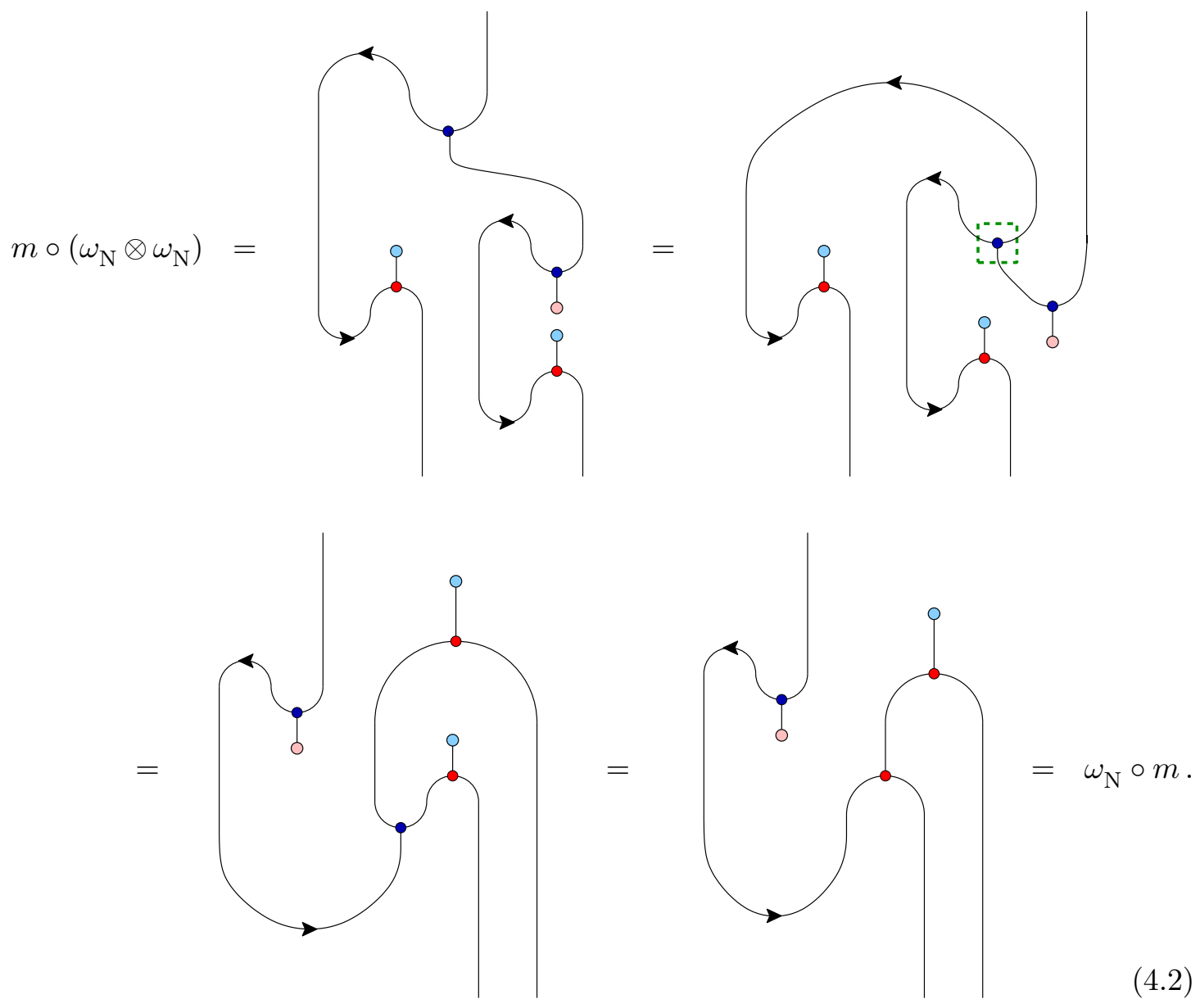

Thus $\omega_{\mathrm{N}}$ respects the product. That it also respects the unit, i.e. $\omega_{\mathrm{N}} \circ \eta=\eta$, follows directly by applying the unit property, sovereignty, duality axioms, and the counit property.

In [8], a symmetric Frobenius algebra $A$ in $\mathcal{C}$ is defined as a Frobenius algebra for which the morphisms $\Phi$ and $\tilde{\Phi}$ in (3.6) coincide. Thus $A$ is symmetric iff

$$
\omega_{\mathrm{N}}=i d_{\dot{A}}
$$

If $A$ is symmetric in this sense, then $\Phi=\tilde{\Phi}$ is an isomorphism between $A$ and $A^{\vee}$ as $A$ bimodules; in $\mathcal{V}_{\text {ect }}$ it is this latter property of $A$ that is commonly used as the defining 
property of a symmetric Frobenius algebra. Also, if $A$ is symmetric, then $\varepsilon \circ \Delta$, i.e. the analogue (3.8) of the nondegenerate invariant bilinear form, is symmetric in the sense that

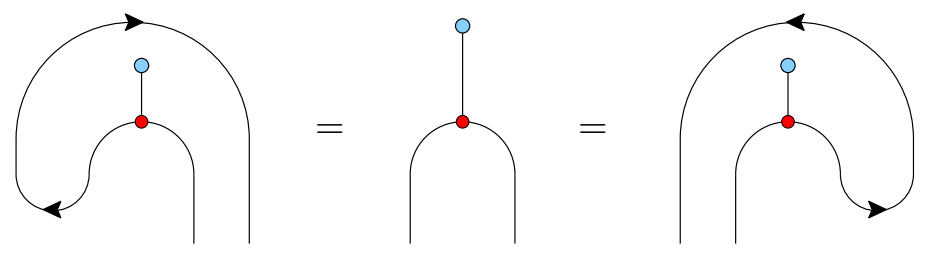

Note that the notion of symmetry relevant here does not involve a braiding. The proper setting setting for Frobenius algebras is the one of monoidal categories, and for symmetric Frobenius algebras the one of sovereign monoidal categories; no braiding is required.

That (4.3) characterizes a symmetric algebra means that $\omega_{\mathrm{N}}$ is a Nakayama automorphism (see e.g. [14, 20]). Any other Nakayama automorphism differs from $\omega_{\mathrm{N}}$ by an inner automorphism, that is, by a morphism of the form

$$
\varpi_{\alpha}=m \circ(\alpha \otimes m) \circ\left(i d_{\dot{A}} \otimes \alpha^{-1}\right)
$$

for some morphism $\alpha \in \operatorname{Hom}(\mathbf{1}, \dot{A})$ that is invertible with respct to the convolution product $\alpha * \beta:=m \circ(\alpha \otimes \beta)$ on $\operatorname{Hom}(\mathbf{1}, \dot{A})$. In other words, one can think of 'the' Nakayama automorphism of $A$ as an element of the $\operatorname{group} \operatorname{Aut}(A) / \operatorname{Inn}(A)$ of outer automorphisms of $A$. In this respect, it is interesting to note that there is an exact sequence

$$
1 \rightarrow \operatorname{Inn}(A) \rightarrow \operatorname{Aut}(A) \rightarrow \operatorname{Pic}\left(\mathcal{C}_{A \mid A}\right)
$$

of groups [11], involving besides the automorphism group and the inner automorphisms the Picard group of the bimodule category $\mathcal{C}_{A \mid A}$. If $A$ is a (Frobenius-) Azumaya algebra $[24,26]$, then $\operatorname{Pic}\left(\mathcal{C}_{A \mid A}\right) \cong \operatorname{Pic}(\mathcal{C})$, so that (4.6) reduces to the Rosenberg-Zelinsky type sequence $1 \rightarrow \operatorname{Inn}(A) \rightarrow \operatorname{Aut}(A) \rightarrow \operatorname{Pic}(\mathcal{C})$ found in [26].

\section{ACKNOWLEDGEMENT}

This work has been supported in part by Vetenskapsrådet under project no. 621-20032385 .

\section{References}

[1] Abrams L, Modules, comodules and cotensor products over Frobenius algebras, $J$. Algebra 219 (1999), 201-213 [math.RA/9806044].

[2] Bakalov B and Kirillov A A, Lectures on Tensor Categories and Modular Functors, Amer. Math. Soc., Providence, 2001.

[3] Curtis C W and Reiner I, Representation Theory of Finite Groups and Associative Algebras, Wiley Interscience, New York, 1962.

[4] Felder G, Fröhlich J, Fuchs J, and Schweigert C, Correlation functions and boundary conditions in RCFT and three-dimensional topology, Compos. Math. 131 (2002), 189237 [hep-th/9912239]. 
[5] Fjelstad J, Fuchs J, Runkel I, and Schweigert C, TFT construction of RCFT correlators V: Proof of modular invariance and factorisation, Preprint hep-th/0503194.

[6] Fröhlich J, Fuchs J, Runkel I, and Schweigert C, Picard groups in rational conformal field theory, Contemp. Math. 391 (2005), 85-100 [math.CT/0411507].

[7] Fröhlich J, Fuchs J, Runkel I, and Schweigert C, Correspondences of ribbon categories, Adv. Math. 199 (2006), 192-329 [math. CT/0309465].

[8] Fuchs J, Runkel I, and Schweigert C, TFT construction of RCFT correlators I: Partition functions, Nucl. Phys. B 646 (2002), 353-497 [hep-th/0204148].

[9] Fuchs J, Runkel I, and Schweigert C, TFT construction of RCFT correlators III: Simple currents, Nucl. Phys. B 694 (2004), 277-353 [hep-th/0403157].

[10] Fuchs J, Runkel I, and Schweigert C, TFT construction of RCFT correlators IV: Structure constants and correlation functions, Nucl. Phys. B 715 (2005), 539-638 [hep-th/0412290].

[11] Fuchs J, Runkel I, and Schweigert C, Ribbon categories and (unoriented) CFT: Frobenius algebras, automorphisms, reversions, Preprint math.CT/0511590.

[12] Joyal A and Street R, The geometry of tensor calculus, I, Adv. Math. 88 (1991), $55-112$.

[13] Kadison L, New Examples of Frobenius Extensions, Amer. Math. Soc., Providence, 1999.

[14] Kadison L and Stolin A A, An approach to Hopf algebras via Frobenius coordinates, Beiträge zur Alg. und Geom. 42 (2001), 359-364 [math.RA/0103001].

[15] Kerler T and Lyubashenko V V, Non-Semisimple Topological Quantum Field Theories for 3-Manifolds with Corners [Lecture Notes in Math. 1765], Springer, New York, 2001.

[16] Kirillov A A and Ostrik V, On a $q$-analog of McKay correspondence and ADE classification of $\widehat{\mathfrak{s l}}(2)$ conformal field theories, Adv. Math. 171 (2002), 183-227 [math.QA/0101219].

[17] Mac Lane S, Categories for the Working Mathematician, Springer, New York, 1971.

[18] Majid S, Braided groups, J. Pure Appl. Alg. 86 (1993), 187-221.

[19] Müger M, From subfactors to categories and topology I. Frobenius algebras in and Morita equivalence of tensor categories, J. Pure Appl. Alg. 180 (2003), 81-157 [math.CT/0111204].

[20] Murray W, Nakayama automorphisms of Frobenius algebras, J. Algebra 269 (2003), 599-609.

[21] Ng S-H and Schauenburg P, Higher Frobenius-Schur indicators for pivotal categories, Preprint math.QA/0503167. 
[22] Ostrik V, Module categories, weak Hopf algebras and modular invariants, Transform. Groups 8 (2003), 177-206 [math.QA/0111139].

[23] Pareigis B, Non-additive ring and module theory I. General theory of monoids, Publ. Math. Debrecen 24 (1977), 189-204.

[24] Pareigis B, Non-additive ring and module theory IV. The Brauer group of a symmetric monoidal category, Springer Lecture Notes in Math. 549 (1976), 112-133.

[25] Szlachányi K, Finite quantum groupoids and inclusions of finite type, Fields Institute Commun. 30 (2001), 393-407 [math.QA/0011036].

[26] Van Oystaeyen F and Zhang Y H, The Brauer group of a braided monoidal category, J. Algebra 202 (1998), 96-128.

[27] Yamagami S, Frobenius algebras in tensor categories and bimodule extensions, Fields Institute Commun. 43 (2004), 551-570. 